\title{
Design and Construction of a Selection Peptide Library Based on the Human Thymic Hormone Thymopoietin Domain
}

\author{
Chunjie Gong, Jianhao Xu, Ling Li, Qianhui Li, Huiling Guo, Li Zheng, \\ Honghao Sun*, and Zhengding Su*
}

The Key Laboratory of Fermentation of the Ministry of Education and Collaborative Innovation Center of Industrial Fermentation, Hubei University of Technology, Wuhan, 430068, China

\section{Introduction}

The activity sites of thymic hormone thymopoietin (TPN) [1] have different biological functions depending on different amino acid sequences, providing a new scaffold for designing peptide inhibitors. In this study, we constructed TPN-based peptide library displayed on the surface of Escherichia coli strain using FimH fusion protein [2]. In principle, the activity site residues of TPN were substituted with randomized peptide sequences and each bacterial cell displayed multiple copies of the same peptide. We checked the bacterial expression on surface of E. coli and the feasibility of the peptide library using a histidine ${ }_{8}$-tag and SDS-PAGE analysis. The histidine ${ }_{8}$ fusion scaffold protein visualized by scanning transmission electron microscopy (STEM) with Ni-NTA-modified magnetic nanoparticles demonstrates that a large fraction of our library expresses to be membrane protein. Furthermore, we proved that the library expressed on the surface of $E$. coli with SDS-PAGE analysis of expression cultures show a large ratio of protein to be insoluble. As expected, the TPN-peptide library was also confirmed to contain $3 \times 10^{4}$ peptides of random 7 -amino acid sequences. The results indicate that many kinds of peptide sequences containing in the TPN scaffold is an efficient technique for screening of peptide drugs.

\section{Results and Discussion}

In this work, we constructed a peptide display library described in Figure 1, and tried to select peptide drugs using the library. We designed the peptide library using the attachment protein FimH of E. coli and human TPN activity site. As for the gene of FimH, it was amplified by polymerase chain reaction (PCR). The gene of TPN was synthesized and integrated with the sequence of FimH by PCR, and cloned into vector pET20b(+). After transformation, the FimH protein was fused with the scaffold

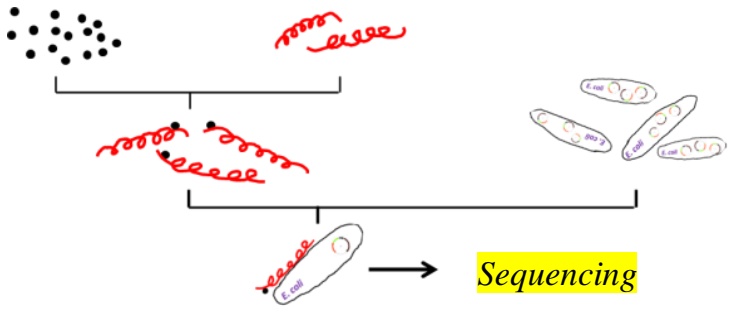

Fig. 1. Schematic showing the design principle of selection peptide library. The peptides were displayed on the surface of E. coli. Then the expressed peptides were selected with target proteins which bind to Ni-NTA-modified magnetic nanoparticles.

SDS-PAGE analysis suggests that a large fraction of His tagged protein was expressed as membrane protein or inclusion body. To prove the expression of membrane protein, we examined the bacterial expression characteristics of this library using Ni-NTA modified magnetic nanoparticles $(5 \mathrm{~nm})$. The expression of the FimH-TPN-His 8 on the surface of $E$. coli was confirmed by scanning transmission electron microscopy. The binding between E. coli carrying plasmid FimH-TPN-His 8 and nanoparticles was observed. The fusion protein with Histidine $_{8}$ expressed on the surface suggested the feasibility of the peptide library. 


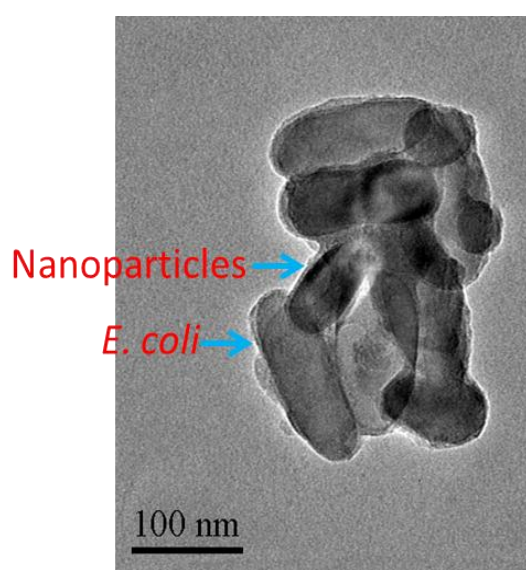

Fig. 2. Nanoparticles bound to E.coli suggests that peptides are expressed on the surface of E. coli.
Based on the vector FimH-TPN containing the BamHI site, we constructed a peptides expression library to generate FimH-TPN-Random using random primers containing random 21-synthetic-nucleotides. The diversity of the library was confirmed by sequencing of thirty clones. The vector FimH-TPN-Random were introduced into recombination E. coli $\mathrm{DH} 5 \alpha$ to prepare random peptide expression library, and stored at $-80^{\circ} \mathrm{C}$ until use.

The peptide display on the surface is a powerful tool for library selection and protein engineering especially using yeast and phage $[3,4]$. In this study, our work is the first time to construct a peptide library using the combination of FimH and TPN. The construction facilitates the display of peptides on the surface of E. coli. We observed a large fraction of insolubility for the proteins in our library. The random peptide expression library was proposed to screen the target proteins. Although the transformation affected the amount of the peptides of the library, we thought it is effective for selection of peptide drugs. Overall, our results demonstrate the design of a peptide library using human TPN activity site and the feasibility of the library for screening of peptide drugs.

\section{Acknowledgments}

This work was supported by the starting fund from Hubei University of Technology.

\section{References}

1. Li, J., et al. Protein Expression and Purification 84, 1-8 (2012), http://dx.doi.org/10.1016/j.pep.2012.04.013

2. Dagmara, I., et al. PLOS Pathogens (2015), http://dx.doi.org/10.1371/journal.ppat.1004857

3. Rhiel, L., et al. PLOS ONE (2014), http://dx.doi.org/10.1371/journal.pone.0114887

4. Clackson, T., et al. Nature 352(6336), 624-628 (1991), http://dx.doi.org/10.1038/352624a0 